\title{
La naturaleza (sonora) de la ciudad. Buildings [New York], de Francisco López ${ }^{*}$
}

\author{
Makis Solomos \\ Université Paris VIII Vincennes - Saint-Denis \\ makis.solomos@univ-paris8.fr
}

\begin{abstract}
Resumen
El artista sonoro Francisco López ha frecuentemente remarcado la importancia de la escucha - "creo que el acto fundamental de composición o creación es escuchar", escribe. A diferencia de la música electroacústica, que elabora su propia materia sonora, sus obras son mayoritariamente salidas del field recording. Sin embargo, no estamos frente a una lógica de documentación o de representación de paisajes sonoros: si bien, con frecuencia, se reconoce el origen de los sonidos, López tiende a privilegiar la inmersión en el sonido en sí. La escucha de sus obras es asíllamada a trabajar sistemáticamente el intervalo entre el sonido como índice y el sonido autonomizado, entre el sonido como dimensión del entorno y el sonido como material artístico. Este intervalo genera formas de subjetivación particulares. El presente texto analizará una obra sonora en donde Francisco López se ha servido de registros realizados en edificios neoyorkinos, dando a escuchar una ciudad hecha de máquinas misteriosas y que parecen en momentos un bosque tropical.
\end{abstract}

Palabras clave

Francisco López, artes sonoras, ecología sonora, escucha.

The (sonant) nature of the city. Buildings [New York] of Francisco López

\begin{abstract}
The sound artist Francisco López has often underlined the importance of listening: "I believe that the most fundamental act of composition or creation is listening", is one of his main statement. While an electroacoustic work elaborates its own sound material, his works are mainly rooted in field recordings. Nevertheless, this has nothing to do with documentation or soundscape representation. Even if very often we can recognize the origins of the sounds, López aims an immersion in sound-itself. Thus, listening to his music means to work constantly with the gap between sound as a sign and sound as an autonomous entity, between sound as an environment's dimension and sound as an artistic material. This gap generates particular forms of subjectivation that this article will explore. This paper will take as example a sound work where Francisco
\end{abstract}

* Recibido: 15 de abril de 2017 / Aceptado: 31 de mayo de 2017. 
Makis Solomos. La naturaleza (sonora) de la ciudad. Buildings [New York]...

López uses recordings that were made in New York buildings, making audible a city made by mysterious machines and sounding sometimes like a rainforest.

Keywords

Francisco Lopez, sound arts, sound ecology, listening.

\section{A natureza (sonora) da cidade. Buildings [Nova York], de Francisco López}

Resumo

O artista de som Francisco López muitas vezes sublinhou a importância de ouvir: "Eu acredito que o ato mais fundamental de composição ou criação está ouvindo", é uma das principais afirmações. Enquanto um trabalho eletroacústico elabora seu próprio material de som, seus trabalhos são principalmente enraizados em gravações de campo. No entanto, isso não tem nada a ver com a documentação ou a representação de paisagem sonora. Mesmo que, muitas vezes, possamos reconhecer as origens dos sons, López visa uma imersão no próprio som. Assim, ouvir sua música significa trabalhar constantemente com a diferença entre o som como sinal e o som como uma entidade autônoma, entre o som como uma dimensão e o som do ambiente como material artístico. Esta lacuna gera formas particulares de subjetivação que este artigo irá explorar. Este artigo tomará como exemplo um trabalho de som onde Francisco López usa gravações feitas em edifícios de Nova York, tornando audible uma cidade feita por máquinas misteriosas e soando às vezes como uma floresta tropical.

Palavras-chave

Francisco López, artes sonoras, ecologia do som, audição. 


\section{Introducción}

Francisco López es con frecuencia calificado ya sea como músico experimental, ya sea como artista sonoro -expresión que él mismo parece preferir ${ }^{1}$ (López, 2003). Pero estaríamos tentados, asimismo, de ubicarlo en la categoría del field recording (grabación de campo), pues lo esencial de sus obras musicales (o sonoras) proviene de registros. Sin embargo, él rechaza la idea de registrar para documentar, testimoniar o representar. Muchos de sus registros han sido realizados en sitios naturales excepcionales - bosques tropicales u otros entornos naturales "salvajes" (wilderness). No es en absoluto trivial indicar que tiene una formación de entomólogo, biólogo y ecólogo y que ha enseñado estas materias en muchas universidades de España y América Latina².

Puede parecer paradójico, tal vez, que Francisco López realice en otros tipos de ambientes sonoros la clase de trabajo que hace con los ambientes naturales, particularmente en los registros de sitios urbanos. Nos interesaremos aquí en Buildings [New York] (2001), creado a partir de registros de edificios neoyorquinos, como lo indica el título, y que forma parte de una trilogía (la Trilogía Americana) cuyos dos otros componentes contienen registros de la wilderness: La Selva (1998) y Wind [Patagonia] (2007). Para permitirnos comprender lo que busca Francisco López en la ciudad, tal como se expone en Buildings [New York], comenzaremos por abordar ciertas cuestiones que promueve su filosofía del sonido.

\section{Wilderness y "naturaleza" urbana: del ambiente sonoro}

La obra de Francisco López rebosa de bosques tropicales: para Belle Confusion 966 (1996), se trata de bosques subtropicales de Brasil, Argentina, Venezuela, Costa Rica, Senegal, Gambia y China; la pieza ya clásica La Selva (1998), que forma parte de la trilogía que aquí nos interesa, utiliza sonidos del bosque tropical (una reserva) del mismo nombre de Costa Rica; Untitled 308 (2013) da a escuchar el bosque tropical de Calakmul (Yucatán, México)... La naturaleza salvaje de Francisco López prescinde de seres humanos ("un mundo vacío de presencia humana" ${ }^{3}$, se lee en las notas del CD de Wind [Patagonia]). Por otro lado, evidentemente, convoca la noción de sublime: "A menudo siento que la naturaleza es un

\footnotetext{
${ }^{1}$ Se declara sonicist (en inglés) más que "músico".

${ }^{2}$ Es difícil obtener en la web o en otra parte informaciones precisas y detalladas sobre este aspecto de Francisco López. En lo que concierne a su formación exacta, tiene, al parecer, un PhD como biólogo ecosistémico (ecosystem biologist). Ver: http://www.franciscolopez.net/field.html.

3 "A world devoid of human presence".
} 
Makis Solomos. La naturaleza (sonora) de la ciudad. Buildings [New York]...

fantasma gigante sin lenguaje y sin el menor interés en mí. Ahí es cuando se convierte en una experiencia emocionante"4 (López, 2001, noviembre).

La presencia de este tipo de naturaleza se explica en gran parte por el trabajo en terreno que López tuvo que realizar como investigador. Como lo explica en una entrevista en el 2000:

He estado haciendo investigación de campo durante los últimos quince años a través de todo el mundo. Esto, obviamente, me da una oportunidad excepcional para experimentar todo tipo de entornos sonoros sorprendentes. [...] La complejidad de los entornos sonoros, su riqueza natural, el inusual ritmo que tiene el flujo de los eventos sonoros -todos estos rasgos han tenido una influencia en mi manera de entender la creación de obras sonoras. Uso cambios radicalmente lentos, dinámicas de nivel extremo (desde los límites de la percepción auditiva hasta el umbral del dolor), un intenso enfoque en los sonidos de banda-ancha y su complejidad-todas las cosas que se encuentra en la realidad sónica de la naturaleza 5 (López, 2002).

Estos propósitos permiten comprender que, en la obra de Francisco López, la naturaleza no estará presente como referencia, símbolo o evocación nostálgica, sino a través de las características sonoras incluso de la música. Estamos cerca del universo de un lannis Xenakis que, en La Légende d'Eer, pone en escena a través del mismo sonido a un auditor que sería un navegador perdido en un océano agitado $^{6}$. Y en estos dos compositores, la naturaleza convocada -la wilderness para uno, la naturaleza caótica para el otro- explica la calidad sonora de sus obras: ellas se orientan hacia el ruido.

Esta naturaleza -salvaje y "bulliciosa" (acústicamente hablando), sino ruidosaes, por definición, no bucólica. Encontramos, así, un desacuerdo profundo entre nuestro compositor y R. Murray Schafer (y, más generalmente, la ecología acústica); desacuerdo que está explicado en el artículo de 1998 "Environmental sound matter":

\footnotetext{
4 "Many times I feel nature as a giant ghost with no language and without the slightest interest on me. That's when it becomes a thrilling experience".

5 "I've been doing research field work in biology for the past 15 years all over the world. This obviously gives me an exceptional chance to experience all sorts of amazing sound environments. [...] The complexity of the sound environments, their natural richness, the unusual pace of the flow of sound events -all these features have influenced my way of understanding the creation of soundworks. I use dramatically slow changes, extreme level dynamics (from the limits of hearing perception to the threshold of pain), an intense focus on broad-band sounds and their complexity-all the things you find in the sonic reality of nature".

6 "Cuando compuse La Légende d'Eer, pensaba en alguien que se encontraría en el medio del Océano. En torno a él, los elementos [...] se agitan" ("Quand j'ai composé La Légende d’Eer, je pensais à quelqu'un qui se trouverait au milieu de l'Océan. Tout autour de lui, les éléments [...] se déchaînent", escribe Xenakis (1995). Sobre Xenakis y la naturaleza, revisar lo planteado por Makis Solomos (2004).
} 
La "afinación"7, básicamente, es un "silenciamiento", como si lo "ruidoso" fuese una condición maligna en sí y, además, un rasgo exclusivo del mundo pos-industrial de influencia humana. [...] Para intentar justificar esta supuesta condición maligna de ciertos ruidos o entornos ruidosos, se usan argumentos relacionales insostenibles, tan pueriles y sorprendentes como, por ejemplo, que "el drone en la música... es un narcótico anti-intelectual" [...] Muchos entornos sonoros naturales son bastante ruidosos (las cataratas, el borde del mar, ciertas selvas tropicales...) y el estado sónico estacionario es un rasgo muy común en la naturaleza (independientemente del carácter ruidoso o silencioso del entorno) ${ }^{8}$ (López, 1998).

Francisco López ama, así, lo que Murray Schafer detesta: paisajes sonoros "lofi", bulliciosos, todo en "confusión" (sonora). "Confusión" (o "Bella confusión") es un título del que a veces se ha servido nuestro artista sonoro. Para Belle confusion 966, hemos visto que los sonidos provienen de muchos bosques tropicales; sin embargo, auditivamente, no se reconoce casi nada, a excepción, a veces, del sonido de la lluvia. Se detectará aquí una de las raíces musicales de López, en las antípodas, de alguna manera, de la ecología acústica: como él mismo lo dice en una entrevista, "uno de mis trabajos recientes, Belle Confusion 969, se creó a partir de grabaciones de los bosques, pese a lo cual suena bastante 'industrial'"9 (López, 1999).

Si muchas obras de Francisco López hacen escuchar ambientes sonoros de la naturaleza, otras lo hacen en relación a ambientes sonoros urbanos. El punto común reside en sus caracteres. En efecto, ellas recogen, en cierta medida, la wilderness de la ciudad, es decir, situaciones y espacios dejados a sí mismos, sin seres humanos: Warszawa Restaurant (1995) reproduce los sonidos de un restaurant vacio; Untitled 119 (2004) utiliza un despegue de avión; Buildings [New York] (2001) hace escuchar máquinas, ascensores y diversos sonidos tan "íntimos" para una ciudad como lo son los sonidos de La Selva para un bosque tropical. "Me siento simplemente tan fascinado por la atmósfera sónica de los túneles del metro y la respiración de los edificios vacíos ${ }^{10}$ como por un bosque tropical", precisa (van Peer, 2002: 15).

\footnotetext{
${ }^{7}$ Referencia al título del libro de Murray Schafer The Tuning of the World... (1977).

8 "The "tuning" is basically a "silencing", as if "noisy" were an evil condition in itself and also an exclusive feature of post-industrial human-influenced world [...] This supposed evil condition of certain noises or noise environments is tried to be justified by untenable relational assertions, as puerile and amazing as, for example, that "the drone in music... is an anti-intellectual narcotic" [...] Many natural sound environments are quite noisy (waterfalls, seashores, certain tropical jungles...) and the sonic steady-state condition is a very common feature in nature (regardless of the noisy or quiet character of the environment)".

9 "One of my recent works, Belle Confusion 969, has been created starting from recordings of forests, yet it sounds pretty 'industrial'".

10 "I'm just as fascinated by the sonic atmosphere of subway tunnels and the breathing of empty buildings".
} 
La obra de López no se opone a estos dos tipos de registros -los de la naturaleza y la ciudad. Los trata de manera idéntica: como largos momentos de inmersión, hechos para ser escuchados profundamente; momentos que el auditor no es invitado a descomponer en sonidos aislados, sino a aprehender globalmente. Es por ello que estos registros no constituyen "paisajes sonoros" según la terminología acústica, sino ambientes sonoros. Si Francisco López prefiere llamar así a los sonidos que nos hace escuchar, es porque uno se sumerge en su interior, en lugar de contemplarlos de frente. Si es lo-fi, es porque el propósito apunta a reforzar la sensación de pertenencia a un medio sonoro, a través de la inmersión, y no a cultivar la contemplación. Ahí donde la noción de "paisaje sonoro" -incluso cuando se la piensa en inglés, lengua que tiene el mérito de emplear una palabra compuesta, soundscape, y que reenvía menos, por consecuente, a la palabra "paisaje"- estará siempre ligada a lo visual y su perspectiva analítica, la noción de ambiente sonoro conviene mejor a la modalidad sintética de la escucha.

\section{La experiencia fenomenológica de lo sonoro}

El hecho de que Francisco López privilegie la percepción sintética (y, así, la escucha) por sobre la percepción analítica explica la diferencia entre sus obras y los magníficos field recordings de un Chris Watson, que están en la búsqueda del "detalle escondido" (Bailey, 2009: 242-263). Del field recording en general -aparte de su crítica a las prácticas más triviales y comerciales, que invitan a escuchar un ambiente sonoro lejano para hacernos viajar gastando poco-, López se desmarca, evitando, muy frecuentemente, documentar sus CDs. Las obras de las que hablamos más arriba, que se han podido vincular a ambientes sonoros (naturales o urbanos) nombrados con precisión, son raras.

Hay idealismo en Francisco López: está del lado de la obra y del sonido en sí. En su crítica del field recording, parte de la constatación de que el registro no puede ser aprehendido como copia de lo real. Ciertamente, nos dice, "la grabación de La Selva no fue modificada o sometida a algún proceso de remezcla mayor o de adiciones. En un contexto tradicional, por tanto, podría decirse que este trabajo tiene entornos sonoros de una naturaleza 'pura'"11, pero no hay que olvidar que, por definición, "un registro no es objetivo, dado que "diferentes micrófonos 'escuchan' de maneras muy diferentes entre si"12 (López, 1998). Y si las mejoras en

\footnotetext{
11 "The recordings of La Selva have not been modified or subjected to any process of further mixing or additions. In a traditional context, it could therefore be said that this work features 'pure' straight nature sound environments".

12 "Different microphones 'hear' so differently".
} 
las tecnologías (por ejemplo, a través de sistemas ambisónicos) dan la ilusión de realismo, se trata, de hecho, de un hiperrealismo donde todo es (re)construido.

En el field recording, la pretensión de hacernos (re)vivir un lugar a través de su ambiente sonoro revela, entonces, la ilusión, esto es, la superchería (hiperrealismo). ¿Qué queda, entonces? Quedan, precisamente, la obra y el sonido "en sí". De esta manera, escuchando La Selva, "lo que puede escucharse en este CD no es La Selva; explícitamente, no pretende serlo. En otras palabras, La Selva (la pieza musical) no es una representación de La Selva (la reserva en Costa Rica)"13 (López, 1998). De esta manera, se escuchan una obra musical y el sonido en sí, lo que explica la referencia a las teorizaciones de la primera música concreta propuestas por Pierre Schaeffer (1952).

Como Schaeffer en su tiempo, López opera una separación entre el sonido -la materia sonora, el ambiente sonoro, la huella registrada...- y su causa. Al igual que Schaeffer, busca, de este modo, una musicalización. Es por ello que rechaza la noción propuesta por Murray Schafer de "esquizofonía", que critica -en nombre de la ecología- la separación entre un sonido y su causa. Según Francisco López, la música nace de la capacidad de la imaginación para escapar de la realidad.

Hemos hablado de idealismo, pero esto no constituye, de hecho, sino una apariencia: se trata sobre todo de una crítica de las posiciones a veces moralizantes de la ecología acústica, en provecho de una defensa de la libertad artística y de la posibilidad, para la obra de arte, de existir en cuanto tal. De hecho, en el fondo, la posición de Francisco López es más bien la de la fenomenología.

En efecto, cuando nos dice que La Selva no es La Selva (la reserva), sino que es la obra sonora así nombrada, no se hace parte de ninguna forma de trascendencia; simplemente, rechaza la idea de representación en virtud de la presencia, aquí y ahora, del sonido. No se trata, por tanto, de representar, sino de ser, nos dicen las obras sonoras de López. Escuchémosle una vez más hablar de la relación entre sus investigaciones científicas y su música:

El trabajar como biólogo me da muchas oportunidades para sumergirme en entornos sonoros sorprendentes, lo que debe tener una influencia en mis concepciones y orientaciones del trabajo con el sonido. No me interesa tanto realizar hallazgos en esta materia, puesto que la estoy viviendo. Lo que me hizo producir La Selva fue un poderoso llamado a sumergirme en tan asombroso asunto sonoro. Contrariamente a las concepciones más difundidas en la materia, en lo que cabe a algunos aspectos esenciales, esto se obtiene mucho mejor mediante el registro que a través del lugar "real". Lo que veo

13 "What you can listen on this CD is not La Selva; it explicitly doesn't pretend to be so. In other words, La Selva (the music piece) is not a representation of La Selva (the reserve in Costa Rica)". 
Makis Solomos. La naturaleza (sonora) de la ciudad. Buildings [New York]...

y siento en La Selva no es esencialmente diferente del resto de mi trabajo. Si sigues el camino que va de la representación al ser, dificílmente podrás notar una diferencia ${ }^{14}$ (van Peer, 2002).

Esta constatación -que se está mejor inmerso en un ambiente sonoro a través de su registro que cuando se está en el lugar de este ambiente- es, sin duda, fundamental: explica, sola, todo el trabajo de López. Se trata de autonomizar la dimensión de lo sonoro, que, por esto, puede ser aislada de su "causa" (el lugar) sin que haya esquizofonía.

Esta autonomización, que vuelve caduco el nivel de representación, permite abrir la escucha a una experiencia fenomenológica de lo sonoro:

No me interesar representar nada específico en mi música. De hecho, tengo un fuerte compromiso con lo contrario; es decir, con desarrollar un mundo sónico tan vacío de significado y propósito, que pueda quedar completamente abierto a la experiencia individual. Un terreno fenomenológico en blanco en el cual cada uno sea obligado a crear y desplazarse ${ }^{15}$ (López, 2000, octubre).

Se trata, en cierta medida, de crear las condiciones para que cada auditor, poniendo entre paréntesis las capas apiladas de significación (que están aglutinadas a los sonidos que escucha o a su subjetividad de auditor), pruebe la experiencia de escuchar como una primera experiencia que pone en contacto directo los sonidos a su subjetividad.

Esta experiencia está ligada a la experiencia del espacio-del espacio aquí y ahora, no del espacio de representación. Es por ello que López precisa que, luego de sus performances, desea que el auditor esté en posición de escuchar el sonido. De ahí la búsqueda, ya evocada, de la inmersión -que permite una nueva comparación con Xenakis ${ }^{16}$. A través de la inmersión, rechazando librarse a la representación y mediante las prácticas de performance que lleva a cabo, López apunta a

\footnotetext{
14 "Working as a biologist provides me with lots of chances for being immersed in amazing sound environments, which must have an influence on my conceptions and directions in my work with sound. I'm not so much interested in finding out about this as I am in living it. What made me produce La Selva was a powerful call to get immersed in such an astonishing sound matter. Contrary to commonly held conceptions, in some essential respects, this is much better attained within the recordings than in the "real" place. What I see and feel in La Selva is not essentially different from the rest of my work. If you move in the path from representation to being, you can hardly see such a difference".

15 "I have no interest in "representing" anything specific with my music. I actually have a strong commitment to do just the opposite; that is, to develop a sonic world that is so devoid of meaning and purpose that it can be completely open for individual experience. A blank phenomenological terrain where everyone is compelled to create and move through".

${ }^{16}$ Cuando se le pregunta si conoce una música instrumental que evoque una aproximación inmersiva, responde: "Por supuesto. Dumitrescu o Xenakis son buenos ejemplos, para mí, de una soprendente aproximación instrumental a la textura, la profundidad y supongo que también al potencial inmersivo de la música
} 
hacer entrar en interacción el cuerpo del auditor (el "cuerpo-oyente") con el espacio concreto, con el lugar en donde éste se encuentra, retomando el propósito de Christine Esclapez (2015). En definitiva, pensando la obra musical como una inmersión sonora propicia a una experiencia fenomenológica, López, lejos de buscar cualquier forma de trascendencia, se hace parte de una escucha aquí y ahora ${ }^{17}$.

\section{De lo sensible y una aproximación ecológica}

“Creo que el acto de composición o creación más fundamental es el de la escucha"18 (López, 2015). "La composición o la performance no son esenciales para la creación musical; la escucha sí lo es. Entender las consecuencias de esto modifica radicalmente nuestro rol y nuestras posibilidades"19 (López, 2001). Se habrá comprendido: la escucha es la operación fundamental para Francisco López. La escucha, es decir, la relación sensible con el sonido.

La escucha buscada por López es una escucha que descontextualiza el sonido, que busca liberarlo de sus referencias, a fin de que pueda apreciarse por sí mismo, según hemos visto. Es por ello, decíamos, que en regla general el compositor da pocas indicaciones acerca de la proveniencia de sus sonidos y de sus intenciones o, si lo hace, agrega siempre que la obra debe ser escuchada sin estas indicaciones. Del mismo modo que -reclamándose del sonido en sí- se refiere a la noción de "objeto sonoro" inventada por Pierre Schaeffer, Francisco López, para calificar la escucha no referencial que desea, reenvía a la idea schaefferiana de "escucha reducida", idea íntimamente ligada a la noción de objeto sonoro. No es trivial recordar que, para definir la escucha reducida, Schaeffer reenvía a Husserl, el fundador de la fenomenología (Solomos, 2013: 184-192). Por "reducción" se entiende la famosa "reducción fenomenológica" (o épochè; o suspensión). Sin reto-

creada". ("Of course. Dumitrescu or Xenakis are good examples for me of an amazing instrumental approach to texture, depth and I guess also immersive potential in the music created" (López, 2010, agosto). La inmersión en la música de Xenakis ha sido tratada por Makis Solomos (2013: 257-261).

${ }^{17}$ Seth Kim-Cohen (2009: 125-148) critica las posiciones -que juzga de "fundamentalistas" y "esencialistas"de un sonido en sí desarrolladas por Francisco López. Según él, siempre hay lenguaje, símbolo. Y si las obras de López son interesantes, esto pasa precisamente a través del "relato" del autor, que consiste en intentar desembarazarse del lenguaje. Esta crítica, como acabamos de verlo, no es pertinente: si López se interroga por el sonido en sí, es para que el auditor pueda realizar la experiencia fenomenológica de lo sonoro y (re)descubrir su escucha - no se trata de una forma de sustanciación del sonido (sustanciación que se encuentra por ejemplo en un Giacinto Scelsi).

18 "I believe that the most fundamental act of composition or creation is listening".

19 "Composition or performance are not essential for music creation; listening is. Understanding the consequences of this makes a radical change in our role and our possibilities". 
mar el vocablo de Schaeffer, López prefiere hablar de escucha "ciega" o de escucha "profunda", expresiones que utiliza indiferentemente (Demers, 2010: 115131).

Es a este tipo de escucha que apuntan las performances de Francisco López. Si hablamos de "performance", más que de concierto, es porque se trata, como el mismo López lo dice, de verdaderas proezas [tour de force] de escucha profunda. Su artículo de 2004 "Against the Stage" ("Contra el escenario") explica por qué rechaza totalmente cualquier otro elemento que el sonoro, se trate del elemento visual o de la presencia escénica. Desea hacer desaparecer la noción de "escena", con el fin de favorecer una escucha multidireccional, como pudieron hacerlo en su momento lannis Xenakis o Luigi Nono; una escucha que refuerza la inmersión y la experiencia fenomenológica.

López retoma la palabra schaefferiana de "acusmática" -escuchar sin ver-que el inventor de la música concreta había sacado de los Pitagóricos. Durante las performances, sumergido en la oscuridad, el auditor escucha, con los ojos vendados -en muchos de sus discos, López recomienda vendarse los ojos incluso en nuestras casas. El hecho de vendar los ojos implica no solamente la imposibilidad de ver, sino también "la cuestión crucial de la entrega a la experiencia. Al ser voluntaria y opcional, la ceguera se convierte, en este contexto, en un instrumento para la escucha transformativa por medio de la aceptación, la sumisión, la dedicación, la confianza, el compromiso. Y si somos aún más resueltos y ambiciosos, se convierte también en una herramienta para la expansión espiritual”20 (López, 2015, noviembre). Durante las performances, López, para transmitir la música, está en el centro; el público se dispone en círculo alrededor de él y en dirección hacia los muros (y no hacia el performer, como se hace en los conciertos clásicos de música acusmática). Se encontrarán en su sitio web fotos de performances y el dispositivo técnico preciso que es requerido. Como testimonian los auditores, en este tipo de performance uno se siente más vulnerable y deviene más sensible (van Peer, 2002: 13).

Para concluir en relación a la filosofía de Francisco López, me gustaría simplemente prolongar la cuestión de la relación sensible al mundo y, más precisamente, a la naturaleza. Hemos visto que, para escuchar (de verdad) un ambiente sonoro, es necesario, justamente, aislar la dimensión propia de lo sonoro y, por consecuente, aislarla de su causa, del lugar donde ha sido registrada. Es así como

\footnotetext{
20 "The crucial question of commitment to the experience. Being voluntary and optional, the blindfold becomes in this context a tool for transformative listening through acceptance, surrendering, dedication, trust, engagement. And if we are even more resolute and ambitious, a tool for spiritual expansion".
} 
podemos librarnos a la experiencia fenomenológica que, poniendo entre paréntesis las significaciones, referencias y asociaciones, nos permite redescubrir el mundo.

La reducción fenomenológica no tiene nada de una operación que termina en un formalismo desechado: hablar de sonido "en si" es abrirse -como lo había hecho Schaeffer- a la morfología sonora; es pasar por la fascinación de la escucha para tomar conciencia de nuestra relación con el mundo. Es en este sentido que la experiencia fenomenológica de lo sonoro puede estar, contrariamente a las apariencias, más en relación con una aproximación ecológica que cualquier soundscape composition (si ésta limita su relación con la naturaleza al simple reconocimiento de la fuente de sus materiales sonoros). Como lo dice López:

La [aproximación] fenomenológica da acceso a un nivel diferente; un nivel menos obvio, menos mundano, más precupado de una aprehensión más profunda de la realidad, más capaz de captar la enormidad de las cosas-en-sí-mismas (por ende, bastante más ecológico que los clichés diletantes que hoy en día escuchamos por doquier). De allí que contrarreste la perpetuación y la imposición del programa de escucha epistemológica como nuestra única vía -para algunos, la única vía posible; para otros, la única vía respetable- de acceso al mundo sónico ${ }^{21}$ (López, 2015, noviembre).

\section{La Trilogía americana y Buildings}

La Trilogía americana comprende tres CDs, ya nombrados: La Selva (1998), BuiIdings [New York] (2001), Wind [Patagonia] (2005). El título Trilogía Americana aparece por primera vez en la cobertura del CD de Wind [Patagonia], en el texto de Christoph Cox: “'Trilogía de las Américas', la obra magna del artista sonoro. Geográficamente, la trilogía cubre el hemisferio occidental, desde la ciudad de Nueva York hasta la punta sur de Argentina. Temporalmente, abarca una buena parte de la carrera de López"22 (Cox, 2007).

En la misma cubierta del disco, dándonos un concentrado de su filosofía, Francisco López escribe:

\footnotetext{
${ }^{21}$ The phenomenological [approach] gives access to a different level; one that is less obvious, less mundane, more concerned with a more profound apprehension of reality, more capable of grasping the enormity of things-in-themselves (hence vastly more ecological than the dilettantist clichés we now hear everywhere). And thus it counteracts the perpetuation and the imposition of the epistemological listening program as our only way-some say the only possible way, some others say the only respectful way-to access the sonic world (López, 2015).

22 "'Trilogy of the Americas', the sound artist's magnum opus. Geographically, the trilogy covers the western hemisphere, from New York City to the southern tip of Argentina. Temporally, it spans a good part of López's career".
} 
Makis Solomos. La naturaleza (sonora) de la ciudad. Buildings [New York]...

Wind [Patagonia] es un trabajo que fue creado en la misma perspectiva que La Selva ([...] que presenta entornos sonoros de la selva de Costa Rica) y Buildings [New York] ([...] que presenta entornos sonoros internos a los edificios de la ciudad de Nueva York). El sonido ambiental no procesado, no remezclado es importante para obtener cierta "realidad". Una valoración de la riqueza y las cualidades esenciales del material sónico original. Una intención no-referencial. Una inmersión fenomenológica extrema, conducida por un anti-racionalismo y un anti-propositivismo. Un mundo vacío de presencia humana. Una pasión por los drones y sus universos internos: esa perceptivamente "invisible" matriz de ruido de banda ancha que fluye constantemente a nuestro alrededor, tanto en la naturaleza como en los entornos hechos por el ser humano. Una proeza de escucha profunda en la que cada auditor debe enfrentar su propia libertad y, de esta manera, $\operatorname{crear}^{23}$ (López, 2007).

Buildings [New York] resulta de un pedido de la asociación Creative Time para un proyecto en y alrededor del Brooklyn Bridge y sus galerías (Brooklyn Bridge Anchorage). El compositor registró edificios neoyorquinos de enero a marzo del 2001. Se escuchan todo tipo de ruidos, que podemos imaginar emanando de oficinas, edificios residenciales y otros inmuebles: ascensores, sistemas de calefacción, salas de máquinas, cables, tubos y conductos de aire, relojes, termostatos, cámaras de video, agua...

Tal como en los registros de la wilderness, no hay, en efecto, presencia humana. Igualmente, el artista sonoro nos da a escuchar sucesiones de pistas (entre 2 y 5 minutos) de registros de momentos particulares, de manera que se le puede seguir a través de su visita en las entrañas de la ciudad. Estos registros son poco retocados en estudio -estamos lejos de las operaciones morfológicas de la música concreta: es el único motivo por el cual se podría decir que Francisco López está más lejos de esta última que de la soundscape composition. En ambos casos, asimismo, se trata ante todo de una experiencia temporal. De ahí la duración, considerable, de las obras (45 min 24 s para La Selva; 1 h 09 min para Buildings [New York]; 56 min 55 s para Wind [Patagonia]): las duraciones largas son más efectivas para situar al auditor en una condición de escucha profunda (López, 2010, agosto). Es por esto que las texturas evolucionan poco o nada, desplegándose durante largos momentos, hasta que nos sumergimos del todo, y terminando por desaparecer (rápida e incluso bruscamente) en beneficio de una nueva textura.

\footnotetext{
23 "Wind [Patagonia] is a work that has been created under the same perspective than La Selva ([...] featuring sound environments from the Costa Rica jungle) and Buildings [New York] ([...] featuring inner sound environments of buildings in New York City). Non-processed, not mixed environmental sound matter from a certain "reality". An appraisal of the richness and essential qualities of the original sonic material. A non-referential intention. An extreme phenomenological immersion led by anti-rationality and anti-purposefulness. A world devoid of human presence. A passion for drones and their inner universe; that perceptually "invisible" matrix of broad-band noise that is constantly flowing around us, both in nature and in man-made environments. A tour de force of profound listening in which every listener has to face his/her own freedom and thus create" (López, 2007).
} 
Con esta expansión temporal y con sus performances acusmáticas, Francisco López crea las condiciones de la experiencia fenomenológica de lo sonoro.

En efecto, tanto con las obras de la wilderness como con los ambientes sonoros de Buildings [New York], somos invitados, a través de la experiencia fenomenológica de lo sonoro, a una inmersión en un ambiente sonoro (y no a la contemplación de un paisaje sonoro). Dominan igualmente, en los dos casos, los ruidos en banda larga, y Buildings [New York] termina con un terrible ruido blanco, que invade casi la totalidad del espectro. Se puede decir que, si con los ambientes sonoros naturales, López nos da a escuchar la wilderness, con Buildings [New York] nos sumerge en el corazón de la ciudad; en aquello que la ciudad tiene de más "íntimo": en ambos casos, nos hace visitar "el interior" del ambiente sonoro ${ }^{24}$.

\section{Referencias}

\section{Bibliografía}

Cox, Christophe (2007). The Sublime and the Sonic Life of Nature. En Francisco López. Winds [Patagonia] (CD). Holanda: V2_Archief.

Bailey, Thomas (2009). MicroBionic: Radical Electronic Music and Sound Art in the 21st Century. Londres: Creation Books.

Demers, Joanna (2010). Listening Through the Noise. The Aesthetics of Experimental Electronic Music. Oxford: Oxford University Press.

Esclapez, Christine (2015) Les "univers locaux" de Francisco López. Images in-temporelles du temps. Revue Filigrane. Musique, esthétique, sciences société (18). Recuperado el 25 de marzo de 2016 de http://revues.mshparisnord.org/filigrane/index.php?id=680.

Kim-Cohen, Seth (2009). In the Blink of an Ear. Toward a Non-Cochlear Sonic Art. New York: Continuum.

López, Francisco (1998). Environmental sound matter. La Selva (Sound environments from a Neotropical rain forest) (CD). Holanda: V2_Archief. Cobertura.

(1999). Interview. Revue et Corrigée, (40), junio. Recuperado el 25 de marzo de 2016 de http://www.franciscolopez.net/int_revue.html.

(2000, octubre). Interview. Montreal Mirror, Canada. Recuperado el 25 de marzo de 2016 de http://www.franciscolopez.net/int_mm.html.

(2001, noviembre). Towards the blur. Francisco López (sitio web). Recuperado el 25 de marzo de 2016 de http://www.franciscolopez.net/aphorisms.html.

\footnotetext{
${ }^{24}$ Para ampliar estas reflexiones, consultar Voegelin (2014) y Demers (2010).
} 
Makis Solomos. La naturaleza (sonora) de la ciudad. Buildings [New York]...

(2003). Interview. Loop, Chile. Recuperado el 25 de marzo de 2016 de http://www.franciscolopez.net/int_loop.html.

(2007). Cobertura. Winds [Patagonia] (CD). Holanda: V2_Archief.

(2010, agosto). Interview. Musique Machine. Recuperado el 25 de marzo de 2016 de http://www.franciscolopez.net/int_musiquemachine.html.

(2015). Fifteen Questions interview with Francisco López. Unfolding from sound. Fifteen Questions. Recuperado el 25 de marzo de 2016 de https://www.15questions.net/interview/fifteen-questions-interview-francisco-lopez/page-1/.

(2015, 9 de septiembre). Expanded Listening: An Interview with Francisco López. SonicField (sitio web). Recuperado el 26 de junio de 2017 de http://sonicfield.org/2015/09/expanded-listening-an-interview-with-francisco-lopez/.

Peer, René van (2002). Waterfall Music. Broad-Band Sound Sources in the music of Francisco López. MusicWorks. Instruments and influences, (82). 10-17.

Schaeffer, Pierre (1952). À la recherche d'une musique concrète. París: Seuil.

Schafer, Murray (1977). The Tuning of the World. A pioneering Exploration into the Past History and Present State of the Most Neglected Aspect of our Environment: The Soundscape. New York: Random House Inc.

Solomos, Makis (2004). Xenakis et la nature? Entre les mathématiques et les sciences de la nature. Musicalia. Annuario internazionale di studi musicologici, (1). 133-146. Recuperado el 20 de junio de 2017 de https://hal.archives-ouvertes.fr/hal01202902.

(2013). De la musique au son. L'émergence du son dans la musique des XXeXXIème siècles. Rennes: Presses Universitaires de Rennes.

Voegelin, Salomé (2014). Sonic Possible Worlds: Hearing the Continuum of Sound. Londres: Bloomsbury.

Xenakis, Iannis (1995). Interview avec Dominique Druhen. lannis Xenakis 2: La légende $d^{\prime} \operatorname{Eer}(\mathrm{CD}) .45$ min 26 s. París: Montaigne.

\section{Discografía}

López, Francisco (1995). Warszawa Restaurant (CD). 1 h 9 min 8 s. Alemania: Trente Oiseaux.

(1996). Belle Confusion 966 (CD). 55 min 53 s. Alemania: Trente Oiseaux. (1998). Belle Confusion 969 (CD). 51 min 32 s. Francia: Sonoris.

(1998). La Selva (Sound environments from a Neotropical rain forest) (CD). $70 \min 49$ s. Holanda: V2_Archief. (2001). Buildings [New York] (CD). 1 h 9 min 4 s. Holanda: V2_Archief. 

dings.

(2004). Untitled 119 (miniCD). 17 min 31 s. Gran Bretaña: Lapili Recor(2007). Wind [Patagonia] (CD). 56 min 55 s. Holanda: V2_Archief.

(2007). Untitled 308 (CD). 42 min $1 \mathrm{~s}$ (parte 1). 33 min $1 \mathrm{~s}$ (parte 2). Gran Bretaña: Very Quiet Records.

Traducción de Gustavo Celedón 